\title{
Trabecular Bone Score Could Not Predict the Bone Mineral Density of Proximal Humerus
}

\author{
Hyeon Jang Jeong ${ }^{1}$, Joong Mo Ahn' ${ }^{2}$, Joo Han Oh' \\ ${ }^{1}$ Department of Orthopaedic Surgery, Seoul National University Bundang Hospital, Seoul National University College of Medicine, Seongnam; \\ ${ }^{2}$ Department of Radiology, Seoul National University Bundang Hospital, Seoul National University College of Medicine, Seongnam, Korea
}

\author{
Corresponding author \\ Joo Han Oh \\ Department of Orthopaedic Surgery, Seoul \\ National University Bundang Hospital, Seoul \\ National University College of Medicine, \\ 82 Gumi-ro 173 beon-gil, Bundang-gu, \\ Seongnam 13620, Korea \\ Tel: +82-31-787-7197 \\ Fax: +82-31-787-4056 \\ E-mail: ohjh1@snu.ac.kr
}

Received: June 23, 2021

Revised: August 7, 2021

Accepted: August 18, 2021
Background: Osteoporosis is an important clinical factor for tendon healing after arthroscopic rotator cuff repair (ARCR). Conventional dual energy X-ray absorptiometry (DXA) of the hip and lumbar spine (LS) does not represent proximal humeral bone mineral density (BMD). Theoretically, direct measurement of the BMD of the proximal humerus is the best method; however, it is not popular and is non-standardized. Therefore, we evaluate whether the trabecular bone score (TBS) using LS DXA would represent proximal humeral BMD. Methods: Conventional hip and LS DXA and proximal humeral BMD were measured in 212 consecutive ARCR patients, and TBS was calculated using LS DXA. Comparative analysis between the affected and contralateral asymptomatic shoulders was done; moreover, correlation analysis was conducted to evaluate the representativity of TBS for proximal humeral BMD. Regression analysis was performed to elucidate the risk factor of intraoperative suture anchor failure (ISAF). Results: BMDs of the affected shoulder were significantly lower than those of the contralateral side (all $P<0.05$ ). TBS failed to present a strong correlation with proximal humeral BMD (correlation coefficients $0.155-0.506$, all $P<0.05$ ), and the BMD of the greater tuberosity (GT) of the proximal humerus was revealed to be a sole risk factor for ISAF (odds ratio, 0.01, $P=0.020$ ). Conclusions: TBS and conventional hip and LS DXA did not represent proximal humeral BMD. Furthermore, among the various radiological measurements, the BMD of the GT was a sole risk factor of ISAF. Therefore, further research for the direct measurement of proximal humeral BMD is mandatory to predict proximal humeral focal osteoporosis.

Key Words: Bone density · Humerus · Osteoporosis · Rotator cuff · Trabecular bone score

\section{INTRODUCTION}

In countries that are entering an aging society, the importance of osteoporosis is increasing, not only from a medical issue but also from a socio-economic point of view. The lifetime risk of a typical osteoporotic fracture has been reported up to $40 \%$.[1] Furthermore, osteoporosis is a spectrum of degenerative changes related to aging process, and it is closely related not only to fracture but also to several degenerative diseases including rotator cuff tear (RCT).[2,3]

$\mathrm{RCT}$ is one of the most common degenerative diseases related to aging process. [4] Therefore, RCT is frequently diagnosed in elderly patients, and as a result, osteoporosis and/or osteopenia was relatively commonly diagnosed in patients with RCT.[5] Moreover, osteoporosis also affects the prognosis of rotator cuff repair.

\section{Mineral Research}

This is an Open Access article distributed under the terms of the Creative Commons Attribution Non-Commercial License (https://creativecommons.org/licenses/by-nc/4.0/) which permits unrestricted non-commercial use, distribution, and reproduction in any medium, provided the origina work is properly cited.

\section{KSBMR}


Lower bone mineral density (BMD) was reported as a risk factor of healing failure after rotator cuff repair.[2,6,7] Furthermore, a previous study presented that healing of repaired rotator cuff could be enhanced with anabolic agent like recombinant human parathyroid hormone which promotes bone formation.[8]

Currently, dual energy X-ray absorptiometry (DXA) of hip and lumbar spine (LS) is the gold standard to diagnose osteoporosis, however, DXA presented wide variation according to measured anatomical site.[9] Moreover, chronic RCT provokes the focal osteoporosis of greater tuberosity (GT) of humerus based on the disuse of affected shoulder and lack of rotator cuff stress over the GT.[10,11] Therefore, estimation of focal osteoporosis of proximal humeri using conventional hip and LS DXA could not guarantee accuracy.[12]

Furthermore, although DXA is the gold standard method of diagnosing osteoporosis, it cannot assess the microstructure of bone. Therefore, trabecular bone score (TBS) was introduced as a new parameter that could quantitatively measure the microstructure of bone.[13] Several previous studies argued that TBS was a more appropriate method to predict the risk of osteoporotic fracture, not only vertebra,[13-16] but also other bones including humerus.[16]

Theoretically, to predict the bone quality of proximal humerus, direct measurement of proximal humeral BMD would be the best method.[12,17] However, measurement of the proximal humeral BMD using DXA and/or quantitative computed tomography is not a popularly used method. Moreover, proximal humeral BMD still has not clinical criteria like T-score of hip and LS DXA, due to absence of normal population data. Therefore, we hypothesized that TBS would more appropriately represent the proximal humeral BMD compared with conventional hip and LS DXA, and TBS would be the predictor of suture anchor failure. Evaluating hypothesis, we compare the correlation between proximal humeral BMD and raw value of hip and LS BMD, T-score of conventional DXA, and TBS, and evaluate the risk factor of suture anchor failure.

\section{METHODS}

Authors retrospectively reviewed the medical records of consecutive 542 patients who underwent arthroscopic rotator cuff repair (ARCR), which was performed by 1 surgeon
(J.H.O.) between 2010 and 2011, under the approval of the Institutional Review Board (IRB) of the senior author's affiliation (IRB no. B-1506/304-103). Of 542 patients of ARCR, 235 patients who measure the proximal humeral BMD using DXA were included, and patients with previous surgical history in ipsilateral shoulder $(\mathrm{N}=7)$, history of fractures in the ipsilateral shoulder (9), RCT was observed only in subscapularis which was attached to lesser tuberosity (7) were excluded from the study to decrease the heterogeneity. Therefore, 212 patients were finally included in the current study. Patients' demographics (age at the operation, sex, hand dominance), surgical factors (size of the torn rotator cuff, presence of failed suture anchor), and radiologic factors (DXA of hip, spine, and proximal humerus) were collected for analysis.

The size of RCT was measured by a single surgeon intraoperatively, and classified according to the Cofield classification.[18] The presence of failed suture anchor was defined as cases in which the suture anchor was pulled out during knot-tying intraoperatively. BMD of hip, LS, and proximal humerus was evaluated using DXA (Lunar Prodigy Advance; GE Lunar, Madison, WI, USA). DXA of proximal humerus was measured in a supine position with the shoulder in $15^{\circ}$ abduction and $30^{\circ}$ external rotation, and the el-

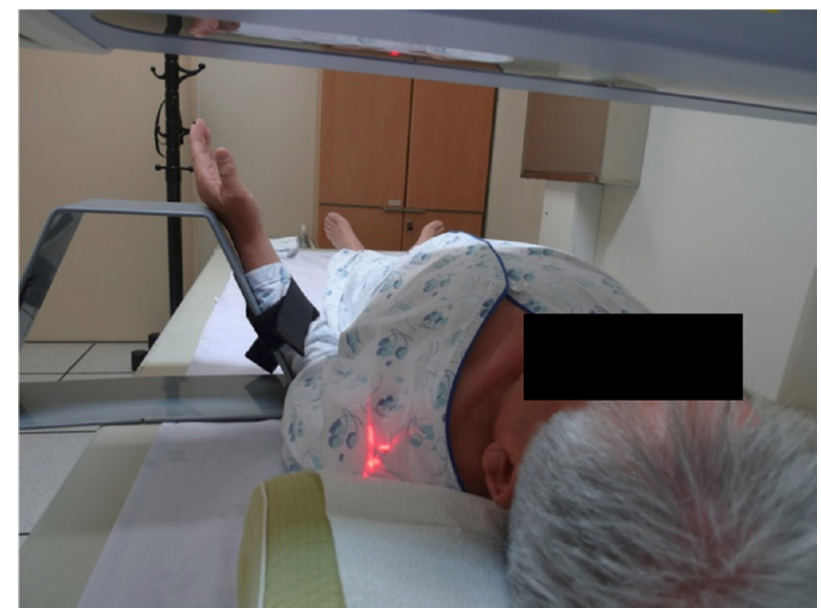

Fig. 1. The position of the arm during the measurement of proximal humeral bone mineral density. Patient lied in supine position with the shoulder in $15^{\circ}$ abduction and $30^{\circ}$ external rotation, and the elbow in a $90^{\circ}$ flexion to prevent the overlapping of the bicipital groove. [Reprinted from "The measurement of bone mineral density of bilateral proximal humeri using DXA in patients with unilateral rotator cuff tear.", by Oh JH, et al., 2014, Osteoporos Int, 25, pp. 2639-48. Copyright 2014 by the Springer International. Reprinted with permission]. 
bow in a $90^{\circ}$ flexion to prevent the overlapping of the bicipital groove (Fig. 1).[12] Region of interest (ROI) of proximal humerus was defined as GT, center of humeral head $(\mathrm{CH})$, and surgical neck (SN) according to a previous study (Fig. 2).[12] T-score was calculated in hip and LS DXA, however, T-score of proximal humerus could not be calculated due to lack of statistical data from normal population. TBS iNsight version 1.9 (Med-Imaps, Geneva, Switzerland) respectively measured the TBS of the 1st to 4th lumbar vertebra (L1, L2, L3, L4), and calculated the mean TBS from L1 to L4 (L1-4), using total spine DXA.

All statistical analyses were conducted using $R$ version 4.0.5 (The R Foundation for Statistical Computing, Vienna, Austria) and RStudio version 1.4.1106 (RStudio Inc., Boston MA, US). Descriptive statistics were used for continuous variables, and the Kolmogorov-Smirnov normality test was

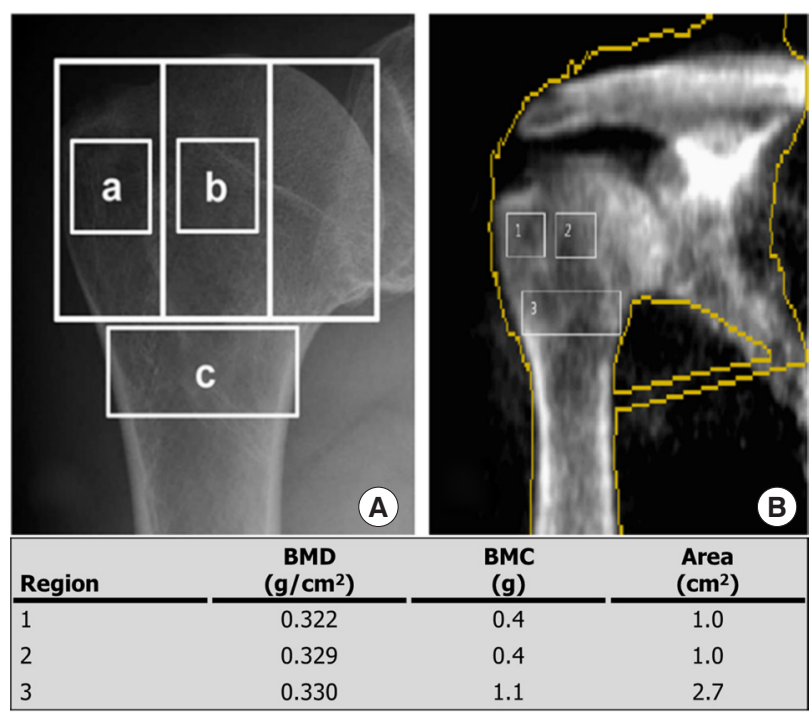

Fig. 2. Region of interest (ROI) of proximal humerus was defined as greater tuberosity (GT), center of humeral head $(\mathrm{CH})$, and surgical neck (SN) and the measurement of the bone mineral density (BMD) using the dual energy $\mathrm{X}$-ray absorptiometry (DXA) in the proximal humerus. (A) To define ROI, proximal humerus was divided into the 3 columns with an even width. In the center of lateral $(a, G T)$ and middle $(b, C H)$ columns, $1 \times 1-\mathrm{cm}^{2}$-sized squares were drawn. ROI of SN (c) was defined as the most common site of involvement in the proximal humeral fracture while creating the block of $1 \mathrm{~cm}$ in height in the metaphysis. (B) The proximal humeral BMD was measured using DXA. [Reprinted from "The measurement of bone mineral density of bilateral proximal humeri using DXA in patients with unilateral rotator cuff tear.", by Oh JH, et al., 2014, Osteoporos Int, 25, pp. 2639-48. Copyright 2014 by the Springer International. Reprinted with permission]. conducted to evaluate the distribution of data composition. Independent $t$-test or Mann-Whitney $\mathrm{U}$ test was conducted for continuous variables, and $\chi^{2}$ or Fisher's exact test was conducted for categorical variables. Paired $t$-test or Wilcoxon signed-rank test was conducted to compare the BMD of affected shoulder and contralateral shoulder. Repeated-measures analysis of variance or Friedman test with Bonferroni correction as post-hoc analysis was used to compare BMD of shoulder according to ROI. Correlation between BMD and TBS was evaluated by Pearson or Spearman correlation analysis. Multi-variable logistic regression analysis was conducted to evaluate the risk factor of intraoperative suture anchor failure (ISAF). All statistical analyses were performed on both sides, and the significance level was set at 0.05 , except post-hoc analysis (set at 0.017 ).

\section{RESULTS}

Ninety-seven male and 115 female patients were included in this study. The mean age at operation was $59.7 \pm 8.4$ years, and female patients were significantly older than male patients ( $P<0.001$; Table 1$)$. RCT was more frequently occurred in hand dominant arm ( $\mathrm{N}=151,71.2 \%)$ than nondominant (ND) arm ( $N=61,28.8 \%)$, and this trend was more pronounced in female patients than male patients $(P=0.003$; Table 1). In terms of tear size, 14 cases (6.6\%) of partial-thickness tear, 26 small tear (12.3\%), 115 medium tear (54.2\%), 18 large tear (8.5\%), and 39 massive tear cases (18.4\%) were presented. Tear size was not statistically different according to sex ( $P=0.487$; Table 1$)$. Osteoporosis was diagnosed in 43 patients $(20.3 \%)$, and it was also more frequently diagnosed in female patients $(P<0.001$; Table 1$)$.

Before further analysis, we compared the BMDs of affected side and contralateral side to evaluate the RCT on BMD.

Table 1. Demographic characteristics according to sex

\begin{tabular}{lccc}
\hline & $\begin{array}{c}\text { Male } \\
(\mathrm{N}=97)\end{array}$ & $\begin{array}{c}\text { Female } \\
(\mathrm{N}=115)\end{array}$ & $P$-value \\
\hline Age (yr) & $57.5 \pm 8.0$ & $61.6 \pm 8.2$ & $<0.001^{\mathrm{a})}$ \\
Hand dominance (D:ND) & $59: 38$ & $92: 23$ & $0.003^{\mathrm{a})}$ \\
$\begin{array}{l}\text { Tear size } \\
\text { (PT:Sm:Md:Lg:Ms) }\end{array}$ & $8: 13: 52: 5: 19$ & $6: 13: 63: 13: 20$ & 0.487 \\
Osteoporosis (Yes:No) & $4: 93$ & $39: 76$ & $<0.001^{\mathrm{a})}$ \\
\hline
\end{tabular}

The data is presented as mean \pm standard deviation or ratio.

a) Statistically significant.

$D$, dominant hand; ND, non-dominant hand; PT, partial thickness tear; Sm, small tear; Md, medium tear; Lg, large tear; Ms, massive tear. 
BMDs of affected side were significantly lower than those of contralateral side in all regions of interests (all $P<0.05$; Table 2). Moreover, BMD of shoulder was significantly different according to ROI in both affected and asymptomatic contralateral side (all $P<0.05$; Table 2), and post-hoc analysis revealed that BMD of $S N$ was significantly higher than BMD of GT and/or CH (all $P<0.017$ ). However, BMD of GT and $\mathrm{CH}$ were not significantly different in both affected side $(P=0.801)$ and contralateral side $(P=0.175)$. Since the BMDs of both sides were different, the subsequent analysis used only the data of affected shoulder.

Table 2. Comparative analysis of bone mineral density of proximal humerus according to rotator cuff tear

\begin{tabular}{lccc}
\hline \multirow{2}{*}{ Site } & \multicolumn{2}{c}{$B M D\left(\mathrm{~g} / \mathrm{cm}^{2}\right)$} & \\
\cline { 2 - 3 } & $\begin{array}{c}\text { Affected side } \\
(\mathrm{N}=212)\end{array}$ & $\begin{array}{c}\text { Contralateral side } \\
(\mathrm{N}=212)\end{array}$ & P-value \\
\hline $\mathrm{CH}$ & $0.425 \pm 0.121$ & $0.451 \pm 0.111$ & $<0.001^{\mathrm{a})}$ \\
GT & $0.424 \pm 0.133$ & $0.459 \pm 0.123$ & $<0.001^{\mathrm{a})}$ \\
SN & $0.526 \pm 0.170$ & $0.547 \pm 0.152$ & $<0.001^{\mathrm{a})}$ \\
$P$ P-value & $<0.001^{\text {a }}$ & $\left.<0.001^{\mathrm{a}}\right)$ & \\
\hline
\end{tabular}

The data is presented as mean \pm standard deviation.

a) Statistically significant.

$\mathrm{BMD}$, bone mineral density; $\mathrm{CH}$, center of humeral head; GT, greater tuberosity; SN, surgical neck.

\section{Osteoporosis, BMD, and TBS}

The lowest T-score of osteoporosis group was $-3.1 \pm 0.6$, and it was significantly lower than that of normal group $(-0.7 \pm 1.1, P<0.001)$. BMD and TBS (Fig. 3) were also statistically lower in osteoporosis group (all $P<0.05$; Table 3 ). To evaluate the correlation between TBS and other measurements of BMD, correlation analyses were conducted. Although TBS of L1 had the highest correlation with the lowest overall T-score, correlation coefficient was not high as 0.622 ( $P<0.001$; Table 4). TBS and the lowest overall BMD of hip (range of correlation coefficient values [r], 0.346-0.524), spine $(r, 0.186-0.458)$, and shoulder $(r, 0.199-0.417)$ presented weak-to-moderate correlation, respectively (all $P<0.05$; Table 4). Furthermore, TBS and BMD of $\mathrm{CH}(\mathrm{r}, 0.155-0.386)$, GT $(r, 0.219-0.379)$, and SN (r, 0.282-0.506) also presented weak-to-moderate correlation (all $P<0.05$; Table 4).

As the TBS failed to present a strong correlation with shoulder BMD, further correlation analyses were conducted. The lowest BMD of hip presented relatively higher correlation with BMD of affected shoulder ( $r$ vs. CH 0.708 , vs. GT 0.664 , vs. SN 0.778; all $P<0.001$ ), however, the lowest BMD of $L S$ ( $r$ vs. CH 0.554 , vs. GT 0.517 , vs. SN r, 0.653 ; all $P<0.001$ ) presented moderate-to-strong correlation with affected shoulder.

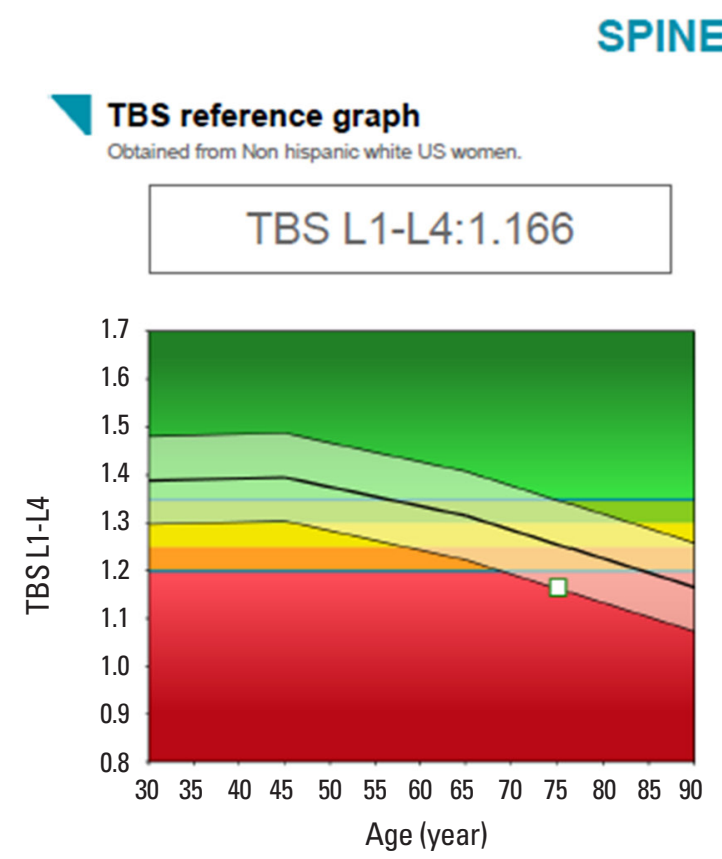

\section{TBS Mapping}

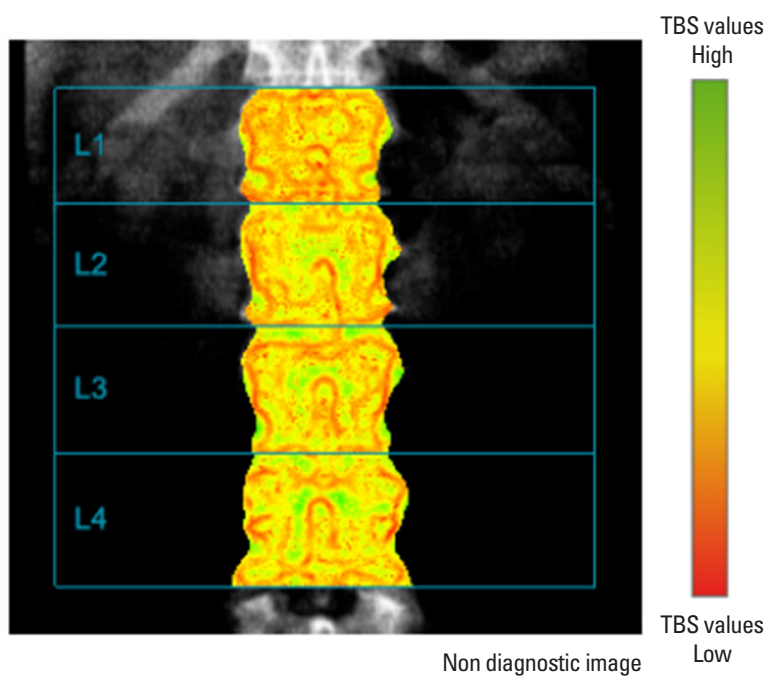

Fig. 3. Trabecular bone score (TBS) of the 1st to 4th lumbar vertebra was measured, and mean TBS from 1st to 4th vertebra was calculated sequentially using lumbar spine dual energy $\mathrm{X}$-ray absorptiometry. 
Table 3. Comparative analysis of bone mineral density according to presence of osteoporosis

\begin{tabular}{|c|c|c|c|}
\hline Region & $\begin{array}{c}\text { Normal } \\
(\mathrm{N}=169)\end{array}$ & $\begin{array}{l}\text { Osteoporosis } \\
(\mathrm{N}=43)\end{array}$ & $P$-value \\
\hline \multicolumn{4}{|l|}{ T-score } \\
\hline Overall & $-0.7 \pm 1.1$ & $-3.1 \pm 0.6$ & $<0.001^{\text {a) }}$ \\
\hline Hip & $-0.1 \pm 1.1$ & $-1.9 \pm 0.8$ & $<0.001^{\text {a) }}$ \\
\hline Spine & $-0.4 \pm 1.4$ & $-3.1 \pm 0.7$ & $<0.001^{\text {a) }}$ \\
\hline \multicolumn{4}{|c|}{ BMD, lowest $\left(\mathrm{g} / \mathrm{cm}^{2}\right)$} \\
\hline Hip & $0.821 \pm 0.142$ & $0.605 \pm 0.092$ & $<0.001^{\text {a) }}$ \\
\hline Spine & $1.053 \pm 0.217$ & $0.754 \pm 0.088$ & $<0.001^{\text {a) }}$ \\
\hline \multicolumn{4}{|l|}{ Shoulder } \\
\hline Affected & $0.416 \pm 0.099$ & $0.279 \pm 0.115$ & $<0.001^{\text {a) }}$ \\
\hline Contralateral & $0.440 \pm 0.102$ & $0.328 \pm 0.079$ & $<0.001^{\text {a) }}$ \\
\hline \multicolumn{4}{|c|}{ BMD, shoulder $\left(\mathrm{g} / \mathrm{cm}^{2}\right)$} \\
\hline \multicolumn{4}{|l|}{$\mathrm{CH}$} \\
\hline Affected & $0.451 \pm 0.108$ & $0.322 \pm 0.114$ & $<0.001^{\text {a) }}$ \\
\hline Contralateral & $0.471 \pm 0.106$ & $0.371 \pm 0.094$ & $<0.001^{\text {a) }}$ \\
\hline \multicolumn{4}{|l|}{ GT } \\
\hline Affected & $0.455 \pm 0.117$ & $0.305 \pm 0.128$ & $<0.001^{\text {a) }}$ \\
\hline Contralateral & $0.485 \pm 0.118$ & $0.358 \pm 0.086$ & $<0.001^{\text {a) }}$ \\
\hline \multicolumn{4}{|l|}{ SN } \\
\hline Affected & $0.575 \pm 0.142$ & $0.335 \pm 0.134$ & $<0.001^{\text {a) }}$ \\
\hline Contralateral & $0.589 \pm 0.134$ & $0.382 \pm 0.094$ & $<0.001^{\text {a) }}$ \\
\hline \multicolumn{4}{|l|}{ TBS } \\
\hline L1 & $0.440 \pm 0.102$ & $0.328 \pm 0.079$ & $<0.001^{a)}$ \\
\hline L2 & $1.363 \pm 0.104$ & $1.254 \pm 0.108$ & $<0.001^{\text {a) }}$ \\
\hline L3 & $1.427 \pm 0.094$ & $1.348 \pm 0.106$ & $<0.001^{\text {a) }}$ \\
\hline L4 & $1.437 \pm 0.113$ & $1.349 \pm 0.127$ & $<0.001^{\text {a) }}$ \\
\hline L1-4 & $1.377 \pm 0.089$ & $1.266 \pm 0.089$ & $<0.001^{\text {a) }}$ \\
\hline
\end{tabular}

The data is presented as mean \pm standard deviation.

a) Statistically significant.

$\mathrm{BMD}$, bone mineral density; $\mathrm{CH}$, center of humeral head; GT, greater tuberosity; SN, surgical neck; TBS, trabecular bone score.

\section{Subgroup analysis according to presence of failed suture anchor}

To evaluate the risk factors of ISAF (Fig. 4) due to pulled out during knot-tying, subgroup analysis according to presence of failed suture anchor was conducted. Suture anchor was pulled out in 13 patients (6.1\%) intraoperatively. Age, proportion of sex, and tear size were not significantly different according to ISAF (all $P>0.05$; Table 5). Furthermore, presence of osteoporosis was not significantly different in both group ( $P=0.539$; Table 5$)$.

The lowest overall T-score $(P=0.190)$, the lowest T-score of hip ( $P=0.068)$ and spine $(P=0.264)$ were not statistically

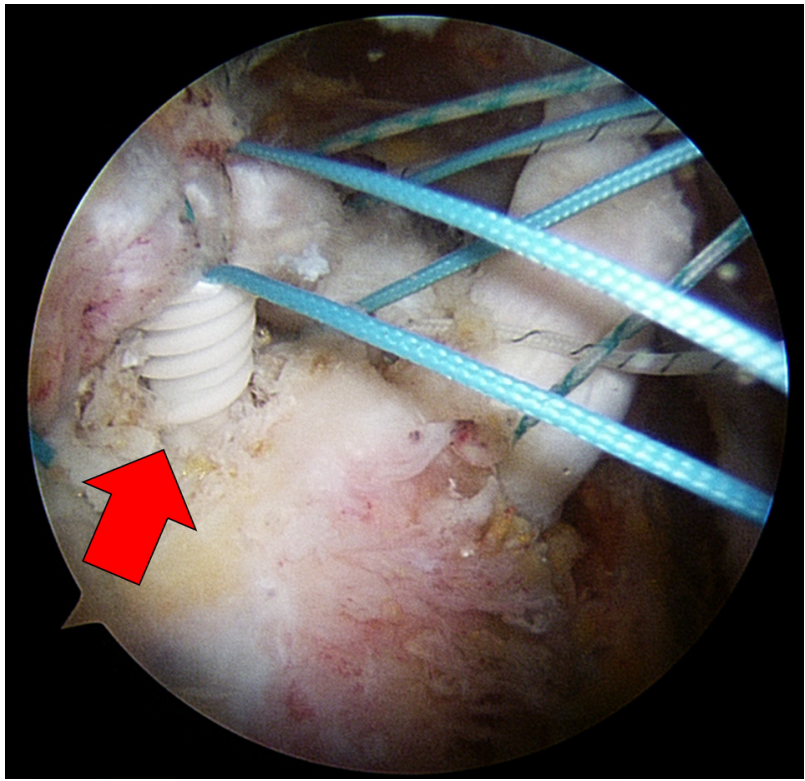

Fig. 4. Arthroscopic finding of intraoperative suture anchor failure (ISAF) is presented. Suture anchor was pulled out during knot-tying (red arrow).

Table 4. Correlation analysis between trabecular bone score and other parameters of bone mineral density

\begin{tabular}{|c|c|c|c|c|c|c|c|}
\hline & T-score (lowest) & \multicolumn{3}{|c|}{ BMD (lowest) } & \multicolumn{3}{|c|}{ BMD (shoulder) } \\
\hline & Overall & Нiр & Spine & Shoulder & $\mathrm{CH}$ & GT & SN \\
\hline \multicolumn{8}{|l|}{ TBS } \\
\hline L1 & $\left.0.622(<0.001)^{a}\right)$ & $0.524(<0.001)^{a)}$ & $0.458(<0.001)^{\mathrm{a})}$ & $0.417(<0.001)^{\mathrm{a})}$ & $0.386(<0.001)^{\mathrm{a})}$ & $0.379(<0.001)^{\mathrm{a})}$ & $0.506(<0.001)^{a}$ \\
\hline L2 & $0.509(<0.001)^{\mathrm{a})}$ & $0.416(<0.001)^{a)}$ & $0.338(<0.001)^{a)}$ & $0.315(<0.001)^{a)}$ & $0.265(<0.001)^{\mathrm{a})}$ & $0.279(<0.001)^{a)}$ & $0.396(<0.001)^{a}$ \\
\hline L3 & $0.354(<0.001)^{a)}$ & $0.346(<0.001)^{a)}$ & $0.240(<0.001)^{a)}$ & $0.199(0.004)^{a)}$ & $0.155(0.020)^{\text {a) }}$ & $0.219(<0.001)^{\text {a) }}$ & $0.282(<0.001)^{a}$ \\
\hline L4 & $0.302(<0.001)^{a)}$ & $0.351(<0.001)^{\mathrm{a})}$ & $0.186(<0.001)^{a)}$ & $0.309(<0.001)^{a)}$ & $0.276(<0.001)^{\mathrm{a})}$ & $0.291(<0.001)^{\mathrm{a})}$ & $0.379(<0.001)^{a}$ \\
\hline L1-4 & $0.531(<0.001)^{\mathrm{a})}$ & $0.485(<0.001)^{\mathrm{a})}$ & $0.355(<0.001)^{\mathrm{a})}$ & $0.371(<0.001)^{\mathrm{a})}$ & $0.325(<0.001)^{\mathrm{a})}$ & $0.348(<0.001)^{\mathrm{a})}$ & $0.466(<0.001)^{a}$ \\
\hline
\end{tabular}

The data is presented as correlation coefficient ( $P$-value).

a) Statistically significant.

BMD, bone mineral density; CH, center of humeral head; GT, greater tuberosity; SN, surgical neck; TBS, trabecular bone score. 
Table 5. Comparative analysis according to presence of failed suture anchor

\begin{tabular}{|c|c|c|c|}
\hline & $\begin{array}{l}\text { Normal } \\
(N=199)\end{array}$ & $\begin{array}{c}\text { ISAF } \\
(N=13)\end{array}$ & $P$-value \\
\hline Age (yr) & $59.6 \pm 8.4$ & $60.7 \pm 8.3$ & 0.656 \\
\hline Sex (male:female) & $93: 106$ & $4: 9$ & 0.405 \\
\hline $\begin{array}{l}\text { Tear size } \\
\text { (PT:Sm:Md:Lg:Ms) }\end{array}$ & 14:24:108:16:37 & $0: 2: 7: 2: 2$ & 0.760 \\
\hline Osteoporosis (Yes:No) & $39: 160$ & $4: 9$ & 0.539 \\
\hline \multicolumn{4}{|l|}{ T-score } \\
\hline Overall & $-1.1 \pm 1.4$ & $-1.7 \pm 1.5$ & 0.190 \\
\hline Hip & $-0.4 \pm 1.3$ & $-1.1 \pm 1.1$ & 0.068 \\
\hline Spine & $-0.9 \pm 1.6$ & $-1.3 \pm 2.0$ & 0.264 \\
\hline \multicolumn{4}{|l|}{ BMD, lowest (g/cm²) } \\
\hline Hip & $0.783 \pm 0.158$ & $0.693 \pm 0.146$ & $0.032^{\mathrm{a})}$ \\
\hline Spine & $0.994 \pm 0.230$ & $0.966 \pm 0.260$ & 0.673 \\
\hline Shoulder & $0.393 \pm 0.114$ & $0.314 \pm 0.136$ & $0.027^{\mathrm{a})}$ \\
\hline \multicolumn{4}{|l|}{ BMD, shoulder $\left(\mathrm{g} / \mathrm{cm}^{2}\right)$} \\
\hline $\mathrm{CH}$ & $0.429 \pm 0.120$ & $0.361 \pm 0.136$ & $0.049^{a)}$ \\
\hline GT & $0.430 \pm 0.131$ & $0.340 \pm 0.143$ & $0.021^{\mathrm{a}}$ \\
\hline SN & $0.532 \pm 0.167$ & $0.434 \pm 0.202$ & $0.043^{\mathrm{a})}$ \\
\hline \multicolumn{4}{|l|}{ TBS } \\
\hline L1 & $1.252 \pm 0.129$ & $1.173 \pm 0.136$ & $0.035^{a)}$ \\
\hline L2 & $1.345 \pm 0.109$ & $1.275 \pm 0.158$ & 0.102 \\
\hline L3 & $1.414 \pm 0.100$ & $1.360 \pm 0.103$ & 0.122 \\
\hline L4 & $1.421 \pm 0.121$ & $1.386 \pm 0.119$ & 0.287 \\
\hline L1-4 & $1.358 \pm 0.097$ & $1.298 \pm 0.117$ & $0.035^{\mathrm{a})}$ \\
\hline
\end{tabular}

The data is presented as mean \pm standard deviation or ratio.

a) Statistically significant.

ISAF, intraoperative suture anchor failure; PT, partial thickness tear; Sm, small tear; Md, medium tear; $\mathrm{Lg}$, large tear; Ms, massive tear; BMD, bone mineral density; $\mathrm{CH}$, center of humeral head; GT, greater tuberosity; SN, surgical neck; TBS, trabecular bone score.

different according to suture anchor failure (Table 5). However, the lowest BMD of hip $(P=0.032)$ and shoulder $(P=$ 0.027) were significantly lower in ISAF group (Table 5). ISAF group also presented lower BMD in all 3 ROls of affected shoulder (all $P<0.05$; Table 5). TBS of L1 $(P=0.035)$ and L1-4 $(P=0.035)$ were statistically lower in ISAF group, however, TBS of $L 2, L 3$, and $L 4$ were not different in both group (all $P>0.05$; Table 5).

To evaluate the risk factor of suture anchor failure, we conduct the multi-variable logistic regression analysis with a backward conditional procedure using variables which present different values between 2 groups (the lowest BMD of hip, GT, SN, and TBS L1-4). The lowest BMD of CH and TBS L1 were excluded from analysis to prevent the multi-collinearity problem. According to Hosmer-Leme-
Table 6. Results of univariate and multivariate logistic regression analysis with a backwards conditional procedure

\begin{tabular}{cccccc}
\hline & \multicolumn{2}{c}{ Univariate analysis } & & \multicolumn{2}{c}{ Multivariate analysis } \\
\cline { 2 - 3 } \cline { 5 - 6 } & Crude OR (95\% CI) & $P$-value & & OR (95\% CI) & $P$-value \\
\hline BMD (lowest) & & & & \\
Hip & $0.018(0.000-1.051)$ & 0.053 & & \\
GT & $0.009(0.000-0.474)$ & $0.020^{\text {a) }}$ & & $0.010(0.000-0.488)$ & $0.020^{\text {a) }}$ \\
SN & $0.037(0.001-0.933)$ & $0.045^{\text {al }}$ & & \\
TBS (L1-4) & $0.003(0.000-0.743)$ & $0.039^{\text {a) }}$ & & \\
\hline
\end{tabular}

The data is presented as odds ratio (95\% confidence interval).

a) Statistically significant.

$\mathrm{OR}$, odds ratio; $\mathrm{Cl}$, confidence interval; $\mathrm{BMD}$, bone mineral density; $\mathrm{GT}$, greater tuberosity; SN, surgical neck; TBS, trabecular bone score.

show test $(P=0.568)$, this multi-variable regression analysis model was considered to be suitable, and in this regression analysis, BMD of GT was revealed as sole risk factor of ISAF (odds ratio, $0.010, P=0.020$; Table 6).

\section{DISCUSSION}

In this study, proximal humeral BMD of all 3 ROls was more decreased in affected shoulder than the asymptomatic contralateral shoulder, and BMD of GT and $\mathrm{CH}$ were statistically lower than that of SN. Although all radiological $B M D$ measurements were lower in osteoporosis group which defined using conventional hip and LS DXA, TBS failed to present strong correlation with shoulder BMD. Moreover, BMD of GT was revealed as sole predictor of ISAF failure.

To predict the risk of ISAF failure during ARCR, accurate measurement of proximal humeral BMD maybe the important factor. However, direct measurement of proximal humeral BMD is not a popular method, and there are still lack of evidence to making a clinical criteria like T-score of conventional DXA. Furthermore, there is no consensus on standard measurement methods; evaluation protocols, ROls, and normal range of evaluated value.

Therefore, we focused on the TBS, which is known to represent the microarchitecture of bone better than conventional DXA.[13] TBS is a noninvasive analytical method based upon the pre-taken conventional LS DXA, and it has the advantage that there is no need for additional irradiation for examination. Furthermore, Popp et al. [16] have argued that TBS could be used to predict the risk of osteoporotic fracture not only in spine, but also in other sites including humerus. 
However, TBS was not appropriately represented the proximal humeral BMD in this study, and we considered that there were 2 reasons based upon the general medical conditions and regional lesions of proximal humerus. Wilson et al. [9] have revealed the regional variation in BMD according to measured anatomical sites and have warned the risk of underestimation of osteoporotic fracture in the upper limb when only using the conventional hip and LS DXA. It is consistent with the finding of this study that TBS and raw value of $L S B M D$ have not strong correlations with proximal humeral BMD. Although conventional hip and LS DXA were used to diagnose systemic osteoporosis, BMD of upper limb could be significantly decreased even in patients with osteopenia and/or a normal range of T-score.

Furthermore, shoulder is not weight-bearing joint. The vertebra and lower extremity including hip joint maintains the posture while supporting the body weight, however, upper extremity including shoulder joint does not bear the body weight. Therefore, the bone density of the upper extremities can maintain adequate stress only with tension based on the attached tendons and their movements. According to Wolff's law, detached tendons and disuse of affected shoulder due to pain and disability may provoke the loss of adequate stress on proximal humerus, and ultimately causes focal osteoporosis in RCT.[19,20] This phenomenon was consisted of that the BMDs of affected shoulder was significantly decreased than asymptomatic contralateral shoulder in this study and previous one.[12]

Another subject of this study is to elucidate the predictor for ISAF failure. Previous studies revealed that healing failure was more frequently occurred in patients with lower BMD, $[2,6,7]$ and a similar result was presented in this study. However, evaluation of BMD in previous studies were based on the conventional hip and LS DXA. Considering the results of this study, direct measurement of BMD of GT is the more accurate method that reflects the BMD of suture anchor insertion site.

Furthermore, different BMD of GT according to anatomical location have been reported.[17,21] These studies have presented that the proximal part of the greater tubercle has more dense trabecular BMD at the posterior portion than anterior, $[17,21]$ and time-zero biomechanical studies have reported that the difference of pullout strength according to BMD and location of suture anchor.[22,23]

To the best of the authors' knowledge, this study has the advantage of being the first to evaluate whether the LS TBS would represent the proximal humeral BMD and conduct direct comparison between proximal humeral BMD and other radiological measurement methods including TBS, raw value of hip and LS BMD, and T-score of conventional DXA. Further, this study explained the reason for unexpected ISAF failure in patients with normal BMD which depends on the difference between systemic osteoporosis and focal osteoporosis of proximal humerus.

However, several limitations were remained. Despite the theoretical advantage of quantitative measurement of microarchitecture of trabecular bone, we calculated TBS only in the LS. However, software which used in this study did not guarantee the accuracy in proximal humerus and femur. Therefore, we calculated the TBS only in LS DXA to decrease the bias based on the technical error of software. Furthermore, we do not compare the study group with a young, normal population. However, purpose of this study is to evaluate the correlation between TBS and BMD of proximal humerus, not establish the T-score of proximal humeral BMD. Moreover, to calculate the T-score of proximal humeral BMD, a large-scale study is required to obtain data from the normal population, and it is beyond the scope of this study. As the need for direct measurement of proximal humeral BMD has emerged through this study, we conclude that large-scale studies including young, normal population will be needed to establish an independent T-score of proximal humerus. Last of all, we did not measure the other systemic bone quality indicators including laboratory markers. Although we focused on the focal osteoporosis of proximal humerus in patients with a RCT, systemic bone metabolism may affect the occurrence of ISAF failure. Therefore, further study that evaluate correlation between ISAF and other systemic factors like bone turnover markers will be needed.

\section{CONCLUSIONS}

TBS and conventional hip and LS DXA did not represent the proximal humeral BMD. Furthermore, among the various radiological measurements, BMD of GT was a sole risk factor of ISAF. Therefore, further research for the direct measurement of proximal humeral BMD is mandatory for the prediction of proximal humeral focal osteoporosis. 


\section{DECLARATIONS}

\section{Funding}

This study received a grant from the Korean Society for Bone and Mineral Research (KSBMR-2015-4).

\section{Ethics approval and consent to participate}

This study conformed to the ethical guidelines of the 1975 Declaration of Helsinki and was approved by the Institutional Review Board.

\section{Conflict of interest}

No potential conflict of interest relevant to this article was reported.

\section{ORCID}

Hyeon Jang Jeong https://orcid.org/0000-0002-8406-0750

Joong Mo Ahn https://orcid.org/0000-0002-1157-0020

Joo Han Oh https://orcid.org/0000-0002-4962-3175

\section{REFERENCES}

1. Melton LJ, 3rd, Chrischilles EA, Cooper C, et al. Perspective. How many women have osteoporosis? J Bone Miner Res 1992;7:1005-10. https://doi.org/10.1002/jbmr.5650070902.

2. Chung SW, Oh JH, Gong HS, et al. Factors affecting rotator cuff healing after arthroscopic repair: osteoporosis as one of the independent risk factors. Am J Sports Med 2011;39: 2099-107. https://doi.org/10.1177/0363546511415659.

3. Chen $X$, Giambini $H$, Ben-Abraham E, et al. Effect of bone mineral density on rotator cuff tear: An osteoporotic rabbit model. PLoS One 2015;10:e0139384. https://doi.org/ 10.1371/journal.pone.0139384.

4. Gumina S, Carbone S, Campagna V, et al. The impact of aging on rotator cuff tear size. Musculoskelet Surg 2013;97 Suppl 1:69-72. https://doi.org/10.1007/s12306-013-0263-2.

5. Cotter EJ, Klosterman EL, Winzenried AE, et al. Osteoporosis screening is often indicated but overlooked prior to rotator cuff repair. Arthrosc Sports Med Rehabil 2021;3:e65965. https://doi.org/10.1016/j.asmr.2021.01.002.

6. Chung SW, Kim JY, Kim MH, et al. Arthroscopic repair of massive rotator cuff tears: outcome and analysis of factors associated with healing failure or poor postoperative function. Am J Sports Med 2013;41:1674-83. https://doi.org/10. 1177/0363546513485719.
7. Kwon J, Kim SH, Lee YH, et al. The rotator cuff healing index: A new scoring system to predict rotator cuff healing after surgical repair. Am J Sports Med 2019;47:173-80. https://doi.org/10.1177/0363546518810763.

8. Jeong JW, Kim MJ, Oh HK, et al. The impact of social media on citation rates in coloproctology. Colorectal Dis 2019;21: 1175-82. https://doi.org/10.1111/codi.14719.

9. Wilson J, Bonner TJ, Head M, et al. Variation in bone mineral density by anatomical site in patients with proximal humeral fractures. J Bone Joint Surg Br 2009;91:772-5. https://doi.org/10.1302/0301-620x.91b6.22346.

10. Clavert P, Bouchaïb J, Sommaire C, et al. Does bone density of the greater tuberosity change in patients over 70 ? Orthop Traumatol Surg Res 2014;100:109-11. https://doi. org/10.1016/j.otsr.2013.11.009.

11. Entezari V, Lazarus M. Surgical considerations in managing osteoporosis, osteopenia, and vitamin D deficiency during arthroscopic rotator cuff repair. Orthop Clin North Am 2019;50:233-43. https://doi.org/10.1016/j.ocl.2018.10. 006.

12. Oh JH, Song BW, Kim SH, et al. The measurement of bone mineral density of bilateral proximal humeri using DXA in patients with unilateral rotator cuff tear. Osteoporos Int 2014;25:2639-48. https://doi.org/10.1007/s00198-0142795-1.

13. Silva BC, Leslie WD, Resch $\mathrm{H}$, et al. Trabecular bone score: a noninvasive analytical method based upon the DXA image. J Bone Miner Res 2014;29:518-30. https://doi.org/10. 1002/jbmr.2176.

14. Hans D, Goertzen AL, Krieg MA, et al. Bone microarchitecture assessed by TBS predicts osteoporotic fractures independent of bone density: the Manitoba study. J Bone Miner Res 2011;26:2762-9. https://doi.org/10.1002/jbmr.499.

15. Iki M, Tamaki J, Kadowaki E, et al. Trabecular bone score (TBS) predicts vertebral fractures in Japanese women over 10 years independently of bone density and prevalent vertebral deformity: the Japanese Population-Based Osteoporosis (JPOS) cohort study. J Bone Miner Res 2014;29: 399-407. https://doi.org/10.1002/jbmr.2048.

16. Popp AW, Meer S, Krieg MA, et al. Bone mineral density (BMD) and vertebral trabecular bone score (TBS) for the identification of elderly women at high risk for fracture: the SEMOF cohort study. Eur Spine J 2016;25:3432-8. https: //doi.org/10.1007/s00586-015-4035-6.

17. Oh JH, Song BW, Lee YS. Measurement of volumetric bone 
mineral density in proximal humerus using quantitative computed tomography in patients with unilateral rotator cuff tear. J Shoulder Elbow Surg 2014;23:993-1002. https:// doi.org/10.1016/j.jse.2013.09.024.

18. DeOrio JK, Cofield RH. Results of a second attempt at surgical repair of a failed initial rotator-cuff repair. J Bone Joint Surg Am 1984;66:563-7.

19. Cadet ER, Hsu JW, Levine WN, et al. The relationship between greater tuberosity osteopenia and the chronicity of rotator cuff tears. J Shoulder Elbow Surg 2008;17:73-7. https://doi.org/10.1016/j.jse.2007.04.017.

20. Waldorff El, Lindner J, Kijek TG, et al. Bone density of the greater tuberosity is decreased in rotator cuff disease with and without full-thickness tears. J Shoulder Elbow Surg 2011;20:904-8. https://doi.org/10.1016/j.jse.2010.12.009.
21. Tingart MJ, Apreleva M, Lehtinen J, et al. Anchor design and bone mineral density affect the pull-out strength of suture anchors in rotator cuff repair: which anchors are best to use in patients with low bone quality? Am J Sports Med 2004;32:1466-73. https://doi.org/10.1177/036354650 3262644.

22. Oh JH, Jeong HJ, Yang SH, et al. Pullout strength of all-suture anchors: Effect of the insertion and traction angle-a biomechanical study. Arthroscopy 2018;34:2784-95. https: //doi.org/10.1016/j.arthro.2018.04.028.

23. Nagra NS, Zargar N, Smith RD, et al. Mechanical properties of all-suture anchors for rotator cuff repair. Bone Joint Res 2017;6:82-9. https://doi.org/10.1302/2046-3758.62.Bjr2016-0225.R1. 
